\title{
Therapeutic Effect Analysis of Radiofrequency Ablation on Transarterial Chemoembolization Postoperative Residual \\ $\mathrm{C} \mathrm{Shi}^{1}, \mathrm{~B} \mathrm{Qiao}^{2}, \mathrm{Q} \mathrm{Yang}^{1}, \mathrm{~B}^{\mathrm{Zheng}}{ }^{1}, \mathrm{X} \mathrm{Yu}^{1}, \mathrm{~S} \mathrm{Wang}^{1}$
}

\begin{abstract}
Objective: To evaluate the clinical effects of radiofrequency ablation (RFA) for transarterial chemoembolization (TACE) postoperative residual tumor in liver cancer.

Methods: A retrospective analysis was performed involving patients with a diagnosis of primary liver cancer who received interventional therapy in the Invasive Technology Department of the Third Affiliated Hospital of Wenzhou Medical University from January 1, 2010 to January 1, 2013. The patients receiving only TACE treatment (43 cases) comprised the TACE group, and patients who received TACE and RFA combined treatment (31 cases) made up the combined treatment group. A comparative analysis assessing therapeutic effect, progression-free survival (PFS), overall survival (OS) and adverse reactions was conducted between the two groups.
\end{abstract}

Results: The objective remission rate of the combined treatment group (87.1\%) was higher than that of the TACE group (65.1\%; $\mathrm{P}<0.05)$. The median PFS (19 months) and median OS (33 months) of the combined treatment group were higher than those of the TACE group (median PFS 14.5 months and median OS 29 months; both $\mathrm{P}<0.05)$. The incidence rate of adverse reactions, such as albumin decline and total bilirubin increase, was lower in the combined treatment group than that in the TACE group $(\mathrm{P}<0.05)$.

Conclusion: RFA can efficiently clear the TACE postoperative residual tumor and prolong both PFS and OS.

Keywords: Hepatic arterial chemoembolization, primary liver cancer, radiofrequency ablation, residual tumour

From: ${ }^{1}$ Department of Intervention Radiology, Third Affiliated Hospital, Wenzhou Medical University, Ruian 325200, China, ${ }^{2}$ Department of Medical Oncology, Ningling People's Hospital, Ningling 476700, China.

Correspondence: Dr X Yu, 108 Wansong Road, Ruian 325200 China, E-mail: yuxxcn15@126.com. 


\section{INTRODUCTION}

For primary liver cancer (PLC), a malignant tumor with high morbidity and mortality, tumor resection is the best treatment option. However, in most cases, the opportunity for surgical resection has passed by the time the diagnosis is made. Therefore, transarterial chemoembolization (TACE) plays a major clinical role in PLC treatment (1); TACE can significantly increase the two-year survival rate $(2,3)$. In cases of large tumor size, ectopic blood supply (the inferior phrenic artery or a branch of the superior mesenteric artery) and arteriovenous fistula (4), embolic agents are not able to affect the whole tumor or embolic agent outflow, which results in only a partial interruption of the blood supply and residual tumor tissue in the first application of TACE. Therefore, repeat TACE or radiofrequency ablation (RFA) is usually required for further treatment.

In addition to surgical resection and TACE, RFA plays an important role in the treatment of liver cancer. With the development of needle electrodes in clinical practice, RFA treatment of liver cancer has gained acceptance (5). In some reports of small liver cancers (diameter $<3.0 \mathrm{~cm}$ ), the therapeutic effectiveness of RFA could approach that of surgical resection (6); however, due to adverse effects, RFA treatment for larger liver cancers (diameter $>5.0 \mathrm{~cm}$ ) has not been accepted (7). In a study of the combined TACE \& RFA treatment of 487 cases of primary liver cancer, tumor size was not significantly correlated with the overall survival rate. The prognosis was closely associated with tumor relapse, tumor number, albumin (ALB), prothrombin time (PT) and platelet (PLT) count (8). Another study in a Danish tertiary liver center compared 18 cases of TACE \& RFA combined treatment with 18 cases of TACE treatment alone and found that combined TACE \& RFA treatment provided superior therapeutic effects (9). A meta-analysis of 21 studies with 3,073 cases showed that TACE \& RFA combined treatment could significantly increase both the short-term and long-term survival rates and improve the prognosis (10). Although the clinical value of 
combined TACE \& RFA treatment in primary liver cancer has been accepted, clearance of tumor tissue residual post-TACE intervention has rarely been reported.

In this study, we compared the second TACE and RFA treatment in cases of residual tumor following an initial TACE; a comprehensive evaluation was performed regarding the clinical effects on the residual tumor and the incidence rate of adverse effects after TACE intervention.

\section{METHODS AND MATERIALS}

\section{Clinical case information}

The patients in this study had a diagnosis of primary liver cancer and received interventional therapy at the Invasive Technology Department of the Third Affiliated Hospital, Wenzhou Medical University, from January 1, 2010 through January 1, 2013. Cases were included in the study based on the following criteria: complete follow-up visit materials; no complications or severe concurrent disease; no portal vein tumor embolization or metastatic lesions; residual tumor found after the first TACE treatment; second interventional therapy by TACE or RFA; and no other combined treatment, such as target drug administration or surgery. The 43 patients who received TACE treatment alone comprised the TACE group while the 31 patients who received combined TACE \& RFA treatment made up the combined treatment group.

The Ethics Committee of the Third Affiliated Hospital, Wenzhou Medical University, approved the study. Written informed consent was obtained from all participants.

\section{Treatment strategy}

TACE treatment was performed under the image guidance of digital subtraction angiography (DSA). The location, size, number and blood supply were confirmed based on arteriography with the conduit selectively introduced through the common hepatic artery and the superior 
mesenteric artery. According to the blood supply, a suitable microconduit was chosen, and the blood-feeding artery of the tumor was super-selected. Chemotherapeutic drugs, including fluorouracil $\left(750 \mathrm{mg} / \mathrm{m}^{2}\right)$ and oxaliplatin $\left(60 \mathrm{mg} / \mathrm{m}^{2}\right)$, were slowly infused. Iodipin (dosage based on the tumor size and blood feed) and pirarubicin $\left(20 \mathrm{mg} / \mathrm{m}^{2}\right)$ were mixed into an emulsion and used as the embolic agent in the tumor blood-feeding arteries. Contrast-enhanced CT and MRI were performed within 1 to 2 months ( $41^{\text {st }}$ day as the median time) postoperatively, and TACE and RFA treatments were repeated in cases with residual tumor.

All RFA treatments were performed under CT guidance. CT-based localization helped to confirm the location, size, number and adjacent tissues of the residual tumor, based on which the direction and depth of needle insertion were determined. The needle electrode of a Rita RFA was inserted into the residual tumor, and the needle expansion level and ablation time length were determined based on the size and location of the residual tumor. Multiple procedures were performed as overlap ablation in cases with a large-sized residual tumor. The scope of ablation was controlled within approximately $0.5-1.0 \mathrm{~cm}$ beyond the tumor edge; the insertion pathway was also ablated while withdrawing the needle.

\section{Follow-up visit, observation indicators and evaluation criterion}

Post-discharge follow-up visits were conducted through hospital re-examination or telephone interviews. The follow-up visits continued until the patient's death or until the deadline of August 31,2014. The length of time from the first TACE treatment to the discovery of tumor progression or patient death was considered to be progression-free survival (PFS), and the length of time from the first TACE treatment until patient death or the last follow-up visit was considered to be overall survival (OS). All patients underwent contrast-enhanced CT or MRI imaging in the $1^{\text {st }}$ to $3^{\text {rd }}$ month postoperatively; indicators including routine blood examination, hepatorenal function, and AFP were measured in the 2 weeks before and the 2 
weeks after the TACE and RFA treatment. According to the mRECIST criteria, a therapeutic effects evaluation was performed, including complete remission (CR), partial remission (PR), stable disease (SD) and progression of disease (PD) (11). "CR+PR" was taken as the objective remission rate (ORR), and " $\mathrm{CR}+\mathrm{PR}+\mathrm{SD}$ " was taken as the disease control rate (DCR).

\section{Statistical analysis}

The data were analyzed using SPS 17.0. Comparison of clinical features, ORR, DCR and adverse reaction rates between the TACE and combined treatment groups was completed with a chi-squared test; age comparisons were made using a $t$ test. A log-rank test was used for comparison of PFS and OS between the two groups, and a Kaplan-Meier survivorship curve was constructed. $\mathrm{P}<0.05$ was considered to indicate statistical significance.

\section{RESULTS}

\section{Clinical features of the TACE group and the combined treatment group}

The clinical characteristics of the TACE and combined treatment groups are summarized in Table 1 . The 2 groups included 58 males and 16 females (74 total cases), with a median age of 64 years old (39-78 years old). The age difference between the TACE group and the combined treatment group was not statistically significant $(t=0.791, P=0.432)$. Similarly, the differences in hepatitis history, cirrhosis background, Child-Pugh grading, AFP, tumor number and tumor size between the 2 groups were not statistically significant.

\section{Evaluation of therapeutic effects}

Based on the contrast-enhanced CT, MRI or ultrasound contrast (3) examination within the $1^{\text {st }}$ to $3^{\text {rd }}$ months postoperatively, an mRECIST evaluation of the therapeutic effects was performed (Figure 1). According to a combined analysis with the second imaging 
examination, the second evaluation was performed for the patients with evaluation results of CR, PR and SD. In the TACE group, there were 11 cases of CR, 17 cases of PR, 11 cases of SD, and 4 cases of PD, with an ORR of $65.1 \%$ and DCR of $90.7 \%$. In the combined group, there were 17 cases of CR, 10 cases of PR, 4 cases of SD, and no cases of PD, with an ORR of $87.1 \%$ and DCR of $100 \%$. The difference in ORR between the 2 groups was significant $\left(X^{2}=4.561, P=0.033\right)$.

\section{Survival analysis}

The median PFS of the TACE and combined treatment groups was 14.5 months and 19 months, respectively (Figure 2), which was a statistically significant difference $(\mathrm{X} 2=7.405$, $\mathrm{P}=0.007)$. The median OS of the two groups was 29 months and 33 months, respectively (Figure 3), which was a statistically significant difference $(\mathrm{X} 2=4.660, \mathrm{P}=0.031)$. The survival rates for the $1^{\text {st }}, 2^{\text {nd }}$ and $3^{\text {rd }}$ years in the TACE group were $88.3 \%(38 / 43), 62.8 \%(27 / 43)$, and $23.3 \%$, respectively $(10 / 43)$. The survival rates for the $1^{\text {st }}, 2^{\text {nd }}$ and $3^{\text {rd }}$ years in the combined treatment group were $93.5 \%$ (29/31), 80.6\% (25/31) and 48.4\% (15/31), respectively.

\section{Adverse effects}

The adverse effects rates of the two groups are summarized in Table 2. No significant difference was found between the two groups for the incidence rate of adverse effects, such as stomachache, fever and transaminase elevation. The incidence rates of albumin decrease and total bilirubin increase in the combined treatment group were lower than those of the TACE group; this was a significant difference. No chemotherapy drug-associated sensory nerve change or drug allergy was observed in the TACE group. In the 31 patients of the combined treatment group, no severe RFA-associated complications such as hemopneumothorax, gastrointestinal perforation or gallbladder-heart reflex were observed. 


\section{DISCUSSION}

Primary liver cancer is a common malignancy of the digestive system that has shown a rising trend in global morbidity and mortality. According to the worldwide survival rate data from 1995 to 2009 (12), among the 57 countries and regions included in the investigation, the 5-year survival rate of liver cancer exceeded $20 \%$ only in Mauritius, Argentina, Japan and Taiwan. In over 20 countries and regions, the survival rates were lower than $10 \%$. In China, the rate was $10 \%-15 \%$. The onset of primary liver cancer is often unobservable, with no obvious symptoms in the early disease stage. In most cases, the disease has reached a later stage or has caused distant metastasis by the time a definitive diagnosis is made, which results in a missed opportunity for early treatment. Therefore, comprehensive non-surgical therapy has become the most common strategy in liver cancer treatment. TACE is widely accepted as the first choice of palliative treatment for liver cancer (1).

Nonetheless, TACE alone is not likely to improve the therapeutic outcomes in liver cancer for several reasons. First, the post-TACE residual tumor usually results from a large tumor size, abundant blood supply, abnormal feed blood vessels or an arteriovenous fistula (13). This type of tumor cannot be cleared entirely with the single TACE method. Second, the therapeutic mechanism of hepatic arterial chemoembolization is based on the hemodynamic features of liver cancer, including tumor blood fed by a liver artery, rich blood supply, and iodine accumulation. Therefore, this method could be effective in solid liver tumors with a rich blood supply, but its effects remain indefinite due to factors such as tumor diffusion, tumor metastasis or poor tumor blood supply. Moreover, hepatic arterial chemoembolization increases rates of tumor recrudesce and metastasis (4) because the hypoxic-ischemic microenvironment can facilitate the high expression of hypoxia-inducible factor (HIF-1) after TACE treatment (14). HIF-1, a regulatory transcription factor, induces the production of lactic dehydrogenase (LDH) and vascular endothelial growth factor (VEGF), which not only 
enhance cell tolerance in hypoxia-ischemia but also promote the formation of tumor angiogenesis (15); therefore, increased expression of these proteins increases the risk of tumor recurrence and metastasis $(16,17)$. Finally, hepatic arterial chemoembolization could cause changes in immune cells and the types and number of cytokines, which play roles in promoting tumor cell death and apoptosis and aggravate hepatocyte injury (18). To improve the prognosis, comprehensive treatment based on TACE is required.

In this study, we compared the second TACE treatment and radiofrequency ablation for the patients with residual tumor after the first TACE. The results demonstrated that the ORR of the combined treatment was higher than that of the TACE treatment alone; the PFS and the OS of the combined treatment group were higher than those of the TACE treatment alone. This suggests better clinical efficacy of radiofrequency ablation for residual tumor after TACE. This may be related to the following factors. First, the main tumor-feeding arteries were blocked or partially blocked by TACE, which inhibited the even dispersal of the embolic agent in the residual tumor and subsequently influenced the second TACE treatment effect. Second, the first TACE caused a hypoxic-ischemic microenvironment in the tumor and stimulated the production of VEGF and new vascularization (15). The larger number of new vessels facilitated the outflow of the embolic agent resulting from the incomplete blood vessel endothelium and high permeability, which also influence the therapeutic effect of the second TACE. Third, the advantage of TACE in application is its effect on larger size tumors that could not be ablated by RFA. Fourth, the embolism or partial embolism of the main tumor-feeding arteries could facilitate heat accumulation in RFA treatment (19). Restriction of heat loss enhanced the effects of RFA.

High-incidence RFA-associated adverse effects include stomachache, fever, nausea, vomiting and weakness without severe pain. Analgesia was required in a small proportion of the patients. For patients with fever but no infection, symptomatic treatment prompted 
resolution in 2-3 days. A temporary increase in transaminase occurs in 50\% of cases after RFA; this level usually returns to normal in 1 week. According to some reports, the incidence rate of post-RFA adverse reactions was positively correlated with the size of the ablation target. These adverse effects return to normal in 2 weeks with symptomatic treatment. In this study, severe complications, such as hemopneumothorax, gastrointestinal perforation or gallbladder-heart reflex, were not observed in the 31 patients who received RFA treatment.

This study is limited in that it is a retrospective study with limited case information; a prospective study with a randomized controlled trial was not included here. In the future, a multicenter prospective clinical investigation remains to be performed.

In conclusion, RFA can effectively clear the post-TACE residual tumor efficiently with a low rate of adverse effects. This treatment could potentially prolong PRS and OS in clinical practice.

\section{AUTHORS' NOTE}

The authors declare no conflicts of interest. 


\section{REFERENCES}

1. Roche A. Therapy of HCC--TACE for liver tumor. Hepato-gastroenterology 2001; 48: 3-7.

2. Llovet JM, Bruix J. Systematic review of randomized trials for unresectable hepatocellular carcinoma: chemoembolization improves survival. Hepatol 2003; 37: 429-42.

3. Takayasu K, Arii S, Ikao I, Omata M, Okita K, Ichida T et al. Prospective cohort study of transarterial chemoembolization for unresectable hepatocellular carcinoma in 8510 patients. Gastroenterol 2006; 131: 461-9.

4. Zhou WB, Yuan FJ, Dai Z. Effect of preoperative TACE on pulmonary metastasis of VX_2 carcinoma in the rabbit liver. Acta Universitatis Medictnae Tangji 2004; 33: 328-07.

5. Tateishi R, Shiina S, Teratani T, Obi S, Sato S, Koike Y et al. Percutaneous radiofrequency ablation for hepatocellular carcinoma. Cancer 2005; 103: 1201-9.

6. Buscarini L, Buscarini E, Di Stasi M, et al. Percutaneous radiofrequency ablation of small hepatocellular carcinoma: long-term results. Eur Radiol 2001; 11: 914-21.

7. Yamakado K, Nakatsuka A, Ohmori S, Vallisa D, Quaretti P, Rocca A. Radiofrequency ablation combined with chemoembolization in hepatocellular carcinoma: treatment response based on tumor size and morphology. J Vasc Interv Radiol 2002; 13: 1225-32.

8. Hui X, Huaming W, Weimin A, Wei M, Ruping Q, Bin Y et al. The efficacy of radiofrequency ablation combined with transcatheter arterial chemoembolization for 
primary hepatocellular carcinoma in a cohort of 487 patients. Plos One, 2014; 9: e89081.

9. Bharadwaz A, Bak-Fredslund KP, Villadsen GE. Combination of radiofrequency ablation with transarterial chemoembolization for treatment of hepatocellular carcinoma: experience from a Danish tertiary liver center. Acta Radiol 2015; 2015: 0284185115603246.

10. Wang Y, Deng T, Zeng L, Chen W. Efficacy and safety of radiofrequency ablation and transcatheter arterial chemoembolization for treatment of hepatocellular carcinoma: A meta-analysis. Hepatol Res 2015; 16: 389-97.

11. Lencioni R, Llovet JM. Modified RECIST (mRECIST) assessment for hepatocellular carcinoma. Semin. Liver Dis 2010; 30: 52-60.

12. Batistić I, Stojanovski J. Global surveillance of cancer survival 1995-2009: analysis of individual data for 25676887 patients from 279 population-based registries in 67 countries (CONCORD-2). Lancet 2015; 385: 977-1010.

13. Llovet JM, Real MI, Montaña X, Planas R, Coll S, Aponte J et al. Arterial embolisation or chemoembolisation versus symptomatic treatment in patients with unresectable hepatocellular carcinoma: a randomised controlled trial. Lancet 2002; 359: 1734-9.

14. Kirkwood JM, Butterfield LH, Tarhini AA, Zarour H, Kalinski P, Ferrone S. Immunotherapy of Cancer in 2012. CA Cancer J Clin. 2012; 62: 309-35.

15. Cha ST, Chen PS, Johansson G, Chu CY, Wang MY, Jeng YM et al. MicroRNA-519c suppresses hypoxia-inducible factor-1alpha expression and tumor angiogenesis. 
Cancer Res 2010; 70: 2675-85.

16. Koukourakis MI, Giatromanolaki A, Panteliadou M, Pouliliou SE, Chondrou PS, Mavropoulou S et al. Lactate dehydrogenase 5 isoenzyme overexpression defines resistance of prostate cancer to radiotherapy. Br J Cancer, 2014; 110: $2217-23$

17. Wu XZ, Ma F, Wang XL. Serological diagnostic factors for liver metastasis in patients with colorectal cancer. World J Gastroenterol 2010; 16: 4084-8.

18. Kim MJ, Jang JW, Oh BS, Kwon JH, Chung KW, Jung HS et al. Change in inflammatory cytokine profiles after transarterial chemotherapy in patients with hepatocellular carcinoma. Cytokine 2013; 64: 516-22.

19. Ahmadzadehfar H, And AS, Biersack HJ. Radioembolization for the treatment of unresectable hepatocellular carcinoma. Intech 2013; 2013: 41-62. 
Table 1. Clinical aspects of the TACE group and combined treatment group.

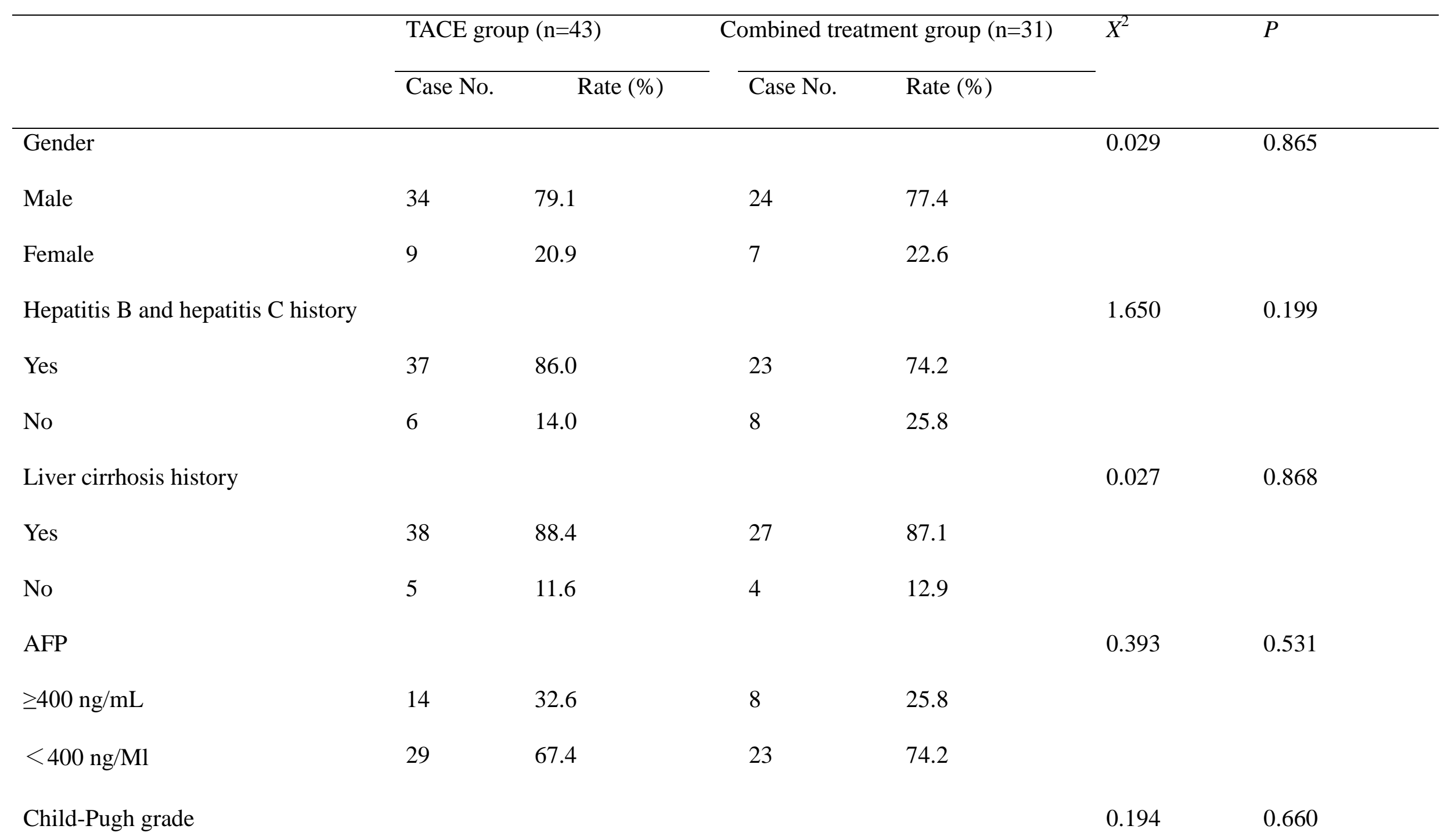


A

B

Tumor No.

Single

Multiple

Tumor size

$\leq 5 \mathrm{~cm}$

$>5 \mathrm{~cm}$
39

90.7

9.3

23

20

16

27

53.5

46.5

37.2

62.8
29

2
93.5

6.5

$0.900 \quad 0.343$

64.5

35.5

$0.538 \quad 0.463$

29.0

71.0 
Table 2. Incidence rate of adverse effects.

\begin{tabular}{|c|c|c|c|c|c|c|}
\hline & \multicolumn{2}{|c|}{ TACE $(n=43)$} & \multicolumn{2}{|c|}{ Combined treatment $(\mathrm{n}=31)$} & \multirow[t]{2}{*}{$X^{2}$} & \multirow[t]{2}{*}{$P$} \\
\hline & Case No. & $\begin{array}{l}\text { Incidence rate } \\
(\%)\end{array}$ & Case No. & Incidence rate $(\%)$ & & \\
\hline \multicolumn{7}{|l|}{ Symptoms } \\
\hline Stomachache & 24 & 55.8 & 19 & 61.3 & 0.473 & 0.492 \\
\hline Fever & 18 & 41.9 & 12 & 38.7 & 0.074 & 0.815 \\
\hline Nausea & 10 & 23.3 & 9 & 29.0 & 0.315 & 0.575 \\
\hline Vomiting & 8 & 18.6 & 6 & 19.4 & 0.007 & 0.935 \\
\hline Weakness & 13 & 30.2 & 6 & 19.4 & 1.117 & 0.291 \\
\hline \multicolumn{7}{|l|}{ Test indicators } \\
\hline ALT rise & 17 & 39.5 & 18 & 58.1 & 2.481 & 0.115 \\
\hline AST rise & 13 & 30.2 & 15 & 48.4 & 2.524 & 0.112 \\
\hline Total bilirubin rise & 4 & 9.3 & 0 & 0 & - & - \\
\hline Albumin decrease & 16 & 37.2 & 3 & 9.7 & 7.155 & 0.007 \\
\hline Leukopenia decrease & 5 & 11.6 & 0 & 0 & - & - \\
\hline
\end{tabular}


RFA on Postoperative Liver Residual Tumour

Thrombocytopenia

Anemia 

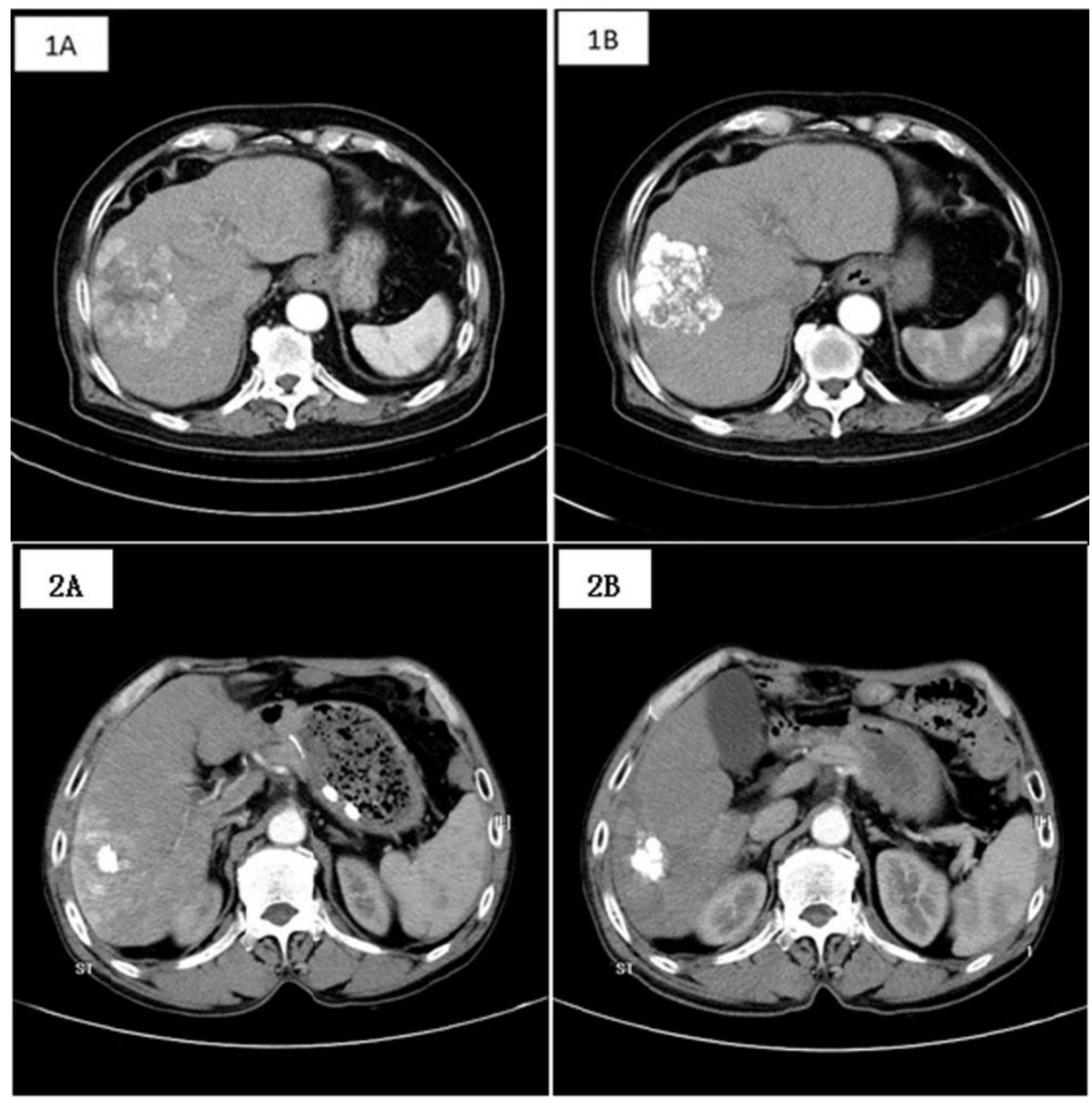

Fig 1: TACE and RFA treatment for the residual after the first TACE. 1A. TACE group, residual tumor after the first TACE. 1B. The same case after the second TACE treatment and entire embolism. 2A. Combined treatment group, residual tumor after the first TACE. 2B. The same case taken after RFA treatment, with entire ablation of the residual. 
RFA on Postoperative Liver Residual Tumour

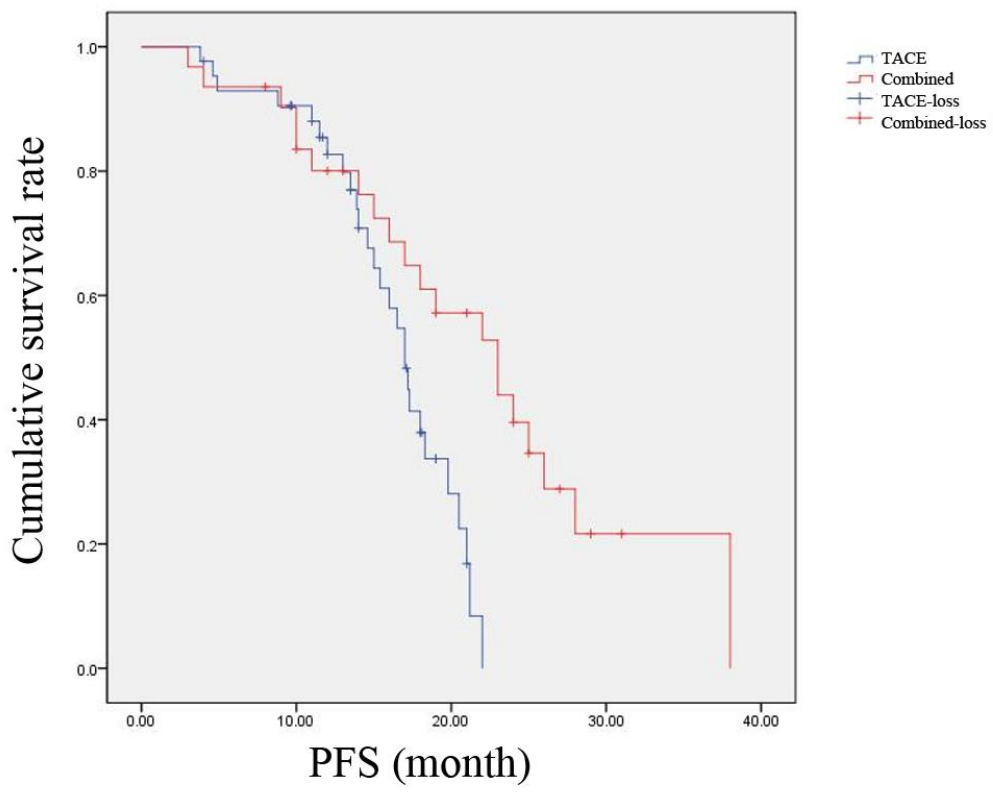

Fig 2: PFS curve of the TACE group and combined treatment group.

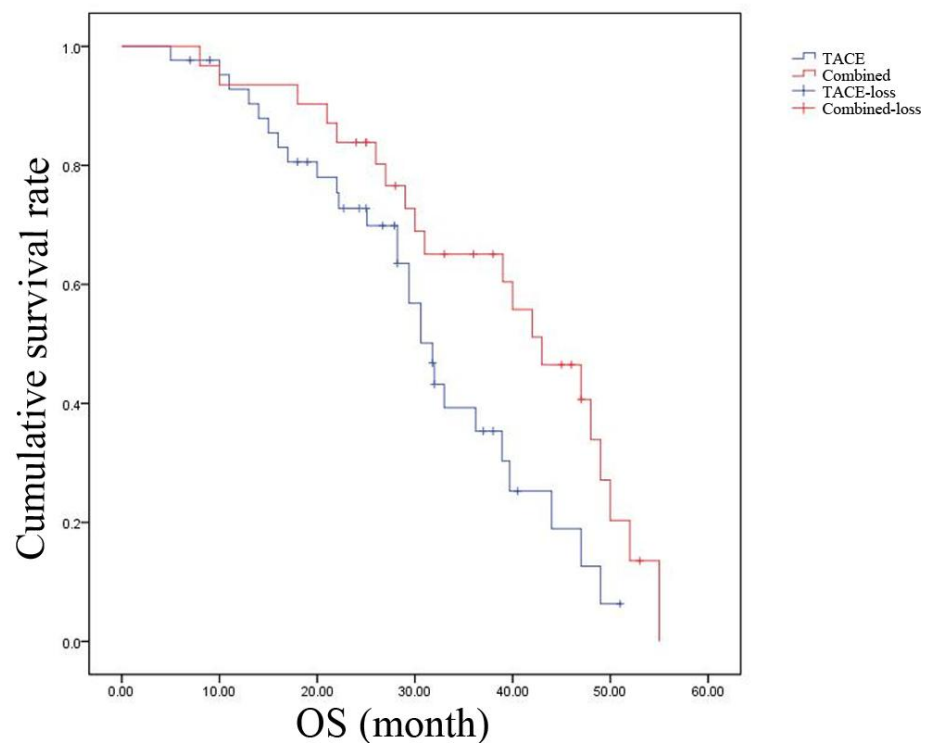

Fig 3: OS curve of the TACE group and combined treatment group. 